\title{
A Study of VO2max in Relation to Body Mass Index before and After Exercise in Healthy Young Adults
}

\author{
Authors \\ Dr Aditya Ghosh ${ }^{1}$, Dr Wasima Jahan ${ }^{2}$ \\ GDMO, Department of Physiology, Professor ${ }^{2}$, Department of Physiology, \\ Assam Medical College, Dibrugarh, Assam, Pin-786002 \\ Email:drrahulchdas@yahoo.com
}

\begin{abstract}
Introduction: VO2max is the maximum capacity to transport \& utilize $O 2$ during incremental exercise and VO2max is the accepted measure of cardiorespiratory effiency. VO2max in normal active male is $38 \mathrm{ml} / \mathrm{kg} / \mathrm{min}$ and in normal active female is $29 \mathrm{ml} / \mathrm{kg} / \mathrm{min}$. Previous studies shows significant lower values of

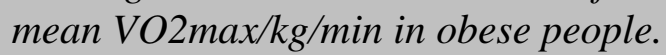

Aims \& Objective: To compare VO2max during tread mill exercise in young healthy adult male in relation to BMI.

Materials and Methods: in the present study 50 healthy young adult male were selected of 18-25 yrs of age. The BMI of the subjects were then measured and their VO2max were calculated before and after 9 mins of treadmill exercise. The formula used to calculate VO2max is VO2max=111.33-(0.42x HR in bpm). RESULT: 50 medical students were selected and they gone through 9 min tread mill test during which heart rate and their VO2max were calculated and then compare with their BMI, which shows a significant negative relationship, with increase in BMI there is decrease in VO2max/kg.

Conclusion: The present study shows that with increase in BMI there is decrease in VO2max/kg, which signifies that increase in BMI leads to decrease in cardiorespiratory fitness.

Abbreviations: HR heart rate, BMI body mass index.
\end{abstract}

\section{Introduction}

Exercise is the body's most common physiologic stress, and it places major demands on the cardiopulmonary system. During exercise different adaptations occur in our body to increase the metabolic rate to 20 times more than the resting level. The most of the adaptations occur in cardiovascular system and respiratory system. The cardiac output increase and these adaptations depend on age, sex, body size and fitness, type of exercise and presence and absence of heart disease. One of the most important factor effected during exercise is total body oxygen uptake. Total body or ventilatory oxygen uptake (VO2) is the amount of oxygen that is extracted from inspired air as the body performs work. The usual measure of the capacity of the body to deliver and utilize oxygen is the maximal oxygen uptake (VO2 max). Thus, the limits of the cardiopulmonary system are historically defined by VO2 max, which can be expressed by the Flick's principle:

$\mathrm{VO} 2$ max $=$ maximal cardiac output $\mathrm{X}$ maximal arterio venous oxygen difference 
Cardiac output must closely match ventilation in order to deliver oxygen to the working muscles. So VO2max depends on two factors one is the cardiac output and another one is the ventilatilatory capacity of the lungs, both of which are effected by exercise. ${ }^{(1)}$

In almost all instances the cost is related positively to body mass; the dependence is high for exercise done against gravity as in walking up an incline or performing stepping exercise, and low for cycle ergometry, where most of the work is done against the ergometer. For any activity the oxygen cost is reduced by familiarity and by training through better coordination and economy of movement. ${ }^{(2)}$ These attributes are affected negatively by increasing age and by ill health. The oxygen cost is increased in obesity. Normal oxygen consumption for a young man at rest is about $250 \mathrm{ml} / \mathrm{min}$. however in maximal condition the oxygen consumption increased to $3600 \mathrm{ml} / \mathrm{min}$ in untrained average male and $4000 \mathrm{ml} / \mathrm{min}$ in athletically trained average male. ${ }^{(3)}$ Both oxygen consumption and total pulmonary ventilation increased about 20 fold between the resting state and maximal intensity exercise in a well trained athlete. ${ }^{(3)}$ BMI is more widely used parameter. In the absence of extra muscle, the range for healthy adults of all ages and both sexes is $18.5-25 \mathrm{~kg} \mathrm{~m}-2$; a BMI in excess of $30 \mathrm{~kg} \mathrm{~m}^{-2}$ is evidence for obesity. It has been found that body size and presence of fat free mass greatly affect the total body oxygen consumption (Vo2max). Exercise testing is one of the best ways to assess ones cardio respiratory fitness when body get expose to extreme stress. The exercise test that is applied should be convenient to the individual and it should be similar to their daily activity. The tread mill is one of the most commonly done and most familiar exercises to every individual. This exercise leads to significant change in cardiopulmonary function of the individuals.

So the present study was done to see the effect of acute exercise on total body oxygen consumption in individuals according to their BMI.

\section{Materials and Method}

50 healthy young students of age group of 18-25yrs are selected from different semester. Subjects with history of cardiopulmonary disease, chronically ill patients, history of surgery or on medication for any illness were excluded from the study. The consent of the students were taken before the test. Weight was recorded to nearest $0.5 \mathrm{~kg}$ with clothing using standard scale. Height was measured to nearest $1 \mathrm{~cm}$ without footwear.BMI Calculated using Quetlet's indices as weight $(\mathrm{kg})$ over height $(\mathrm{m} 2)$. The heart rate was also recorded in resting condition and VO2max was calculated with help of Ardle equation;

$$
\text { VO2max }=111.33-(0.42 x \text { HR in bpm) }
$$

The demonstration of the procedure was given before starting the exercise, and then subjects were asked to walk on the tread mill for 9 mins. The tread mill run under Bruce protocol where speed and inclination increases in every 3 mins interval. The heart rate was recorded from the monitor attached with the tread mill in every 3 mins interval and VO2max was also calculated with the help of Ardle formula.

\section{Results}

The subjects are grouped according to their BMI. They are then divided according to Asian classification of BMI.

Table1: showing the distribution of the population according to their BMI

\begin{tabular}{|l|l|l|l|}
\hline CATEGORY & $\begin{array}{l}\text { Asian } \\
\text { classification } \\
\text { of BMI }\end{array}$ & $\begin{array}{l}\text { No of } \\
\text { students }\end{array}$ & $\begin{array}{l}\text { MEAN } \\
\text { BMI }\left(\mathrm{kg} / \mathrm{m}^{2}\right) \pm \mathrm{SD}\end{array}$ \\
\hline GROUP1 & $18.5-22.9$ & 12 & $22.025 \pm 0.67$ \\
\hline GROUP2 & $23-24.9$ & 14 & $24.390 \pm 0.57$ \\
\hline GROUP3 & $25-29.9$ & 11 & $27.473 \pm 1.13$ \\
\hline GROUP4 & $\geq 30$ & 13 & $33.293 \pm 3.39$ \\
\hline
\end{tabular}


Fig 1: pie diagram showing the distribution of the population according to their BMI

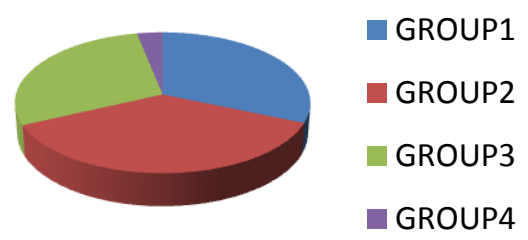

Table 2: The mean VO2max in different groups of the study population during the exercise

\begin{tabular}{|l|c|c|c|c|}
\hline & Group & Group & Group & Group \\
& 1 & 2 & 3 & 4 \\
\hline VO2max & $51.31 \pm$ & $51.51 \pm$ & 63.41 & $61.12 \pm$ \\
1st & 4.85 & 1.86 & \pm 4.08 & 4.21 \\
\hline VO2max & $57.72 \pm$ & $57.12 \pm$ & 55.73 & $52.76 \pm$ \\
2nd & 2.19 & 1.48 & \pm 3.99 & 6.51 \\
\hline VO2max. & $63.07 \pm$ & $63.63 \pm$ & 48.79 & $45.03 \pm$ \\
3rd & 3.13 & 2.07 & \pm 5.17 & 4.97 \\
\hline
\end{tabular}

Fig 2: bar diagram showing the VO2max in different groups in different stages of exercise

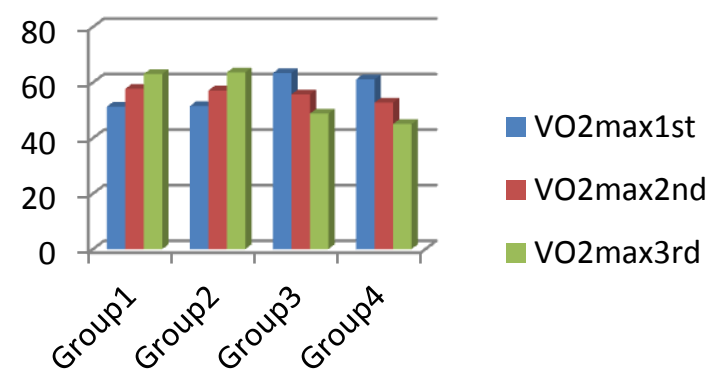

The present study showed a significant negative relationship, with increase in BMI there is decrease in $\mathrm{VO} 2 \mathrm{max}$.

Table 3: showing the $p$ value and $r$ value of VO2max during different stages of BMI

\begin{tabular}{|l|c|c|c|}
\hline & $\begin{array}{c}\text { VO2max } \\
\text { 1sstage }\end{array}$ & $\begin{array}{c}\text { Vo2max } \\
\text { 2nd stage }\end{array}$ & $\begin{array}{c}\text { VO2max } \\
\text { 3rd stage }\end{array}$ \\
\hline$p$ value & 0.13 & 0.00 & -0.00 \\
\hline r value & -0.35 & -0.58 & -0.50 \\
\hline
\end{tabular}

\section{Discussion}

In the present study 50 healthy young adult went through 9 mins of tread mill exercise and their VO2max were calculated during the different stages of exercise. The subjects were divided into four groups according to their BMI and their VO2max were compared. The results showed that there is increase in Vo2max during the exercise which is due to increase cardiac output and increase in ventilation, which is one the body adaptation to acute exercise. In the present study we also found that the subjects having higher BMI had decrease in VO2max during progressive exercise than the persons having BMI within normal range. A negative correlation between $\mathrm{VO} 2 \mathrm{max}$ and $\mathrm{BMI}$ was also found in the following studies:

In the study done by Anjali N. Shete, Smita S Bute, and P.R Deshmukh 'A Study of VO2 Max and Body Fat Percentage in Female Athletes' 25 female athletes of age group 17-22years were selected and VO2 max was determined by Queen's college step test and body fat percentage by skin fold calipers. The mean VO2 max in athletic group was $39.62 \pm$ $2.80 \mathrm{ml} / \mathrm{kg} / \mathrm{min}$. In non-athletic group, VO2 max was $23.54 \pm 3.26 \mathrm{ml} / \mathrm{kg} / \mathrm{min}$. The mean body fat percentage in athletes was $24.11 \pm 1.83 \%$ and in non-athletes it was $29.31 \pm 3.86 \%$. The difference in VO2 max and body fat percentage was statistically significant. $^{(4)}$

In the study done by Suhas Y Shirur, Rajeshwari L and Swathi $\mathrm{H} \mathrm{N}$ 'Effect of increased adiposity on cardiorespiratory fitness of young Indian individuals' VO2max were compared between 30 obese and 30 non-obese subjects aged around 20-25 years. VO2max was estimated by Standard Bruce treadmill test. Various other parameters measured and calculated are weight, height, BMI, heart rate. The results showed that cardiorespiratory fitness was significantly affected $(\mathrm{P}<0.001)$ among obese individuals. $\mathrm{VO} 2 \mathrm{max}$ was significantly lower among obese compared to non obese $(\mathrm{P}<0.001) .{ }^{(5)}$

In a study Prabha Setty, BV Padmanabha, BR Doddamani 'correlation between obesity and cardiorespiratory fittness' 60 young healthy male subjects in the age group of 18 to 22 years were included in this study group. Body mass index was measured as weight in kilograms divided by height in meters square. Cardio respiratory fitness in terms of VO2max was predicted by following the protocol of Treadmill Jogging Test (TMJ). There was a 
highly significant negative correlation between obesity and VO2max, $r=-0.88 \mathrm{p}<0.05$. In contrast, obesity shows a highly significant direct correlation with Treadmill Heart Rate, $r=0.80 \mathrm{p}<0.05$. $^{(6)}$

In the study Assessment of cardiovascular fitness [VO2 max] among medical students by Queens College step test done by Tauseef Nab etal VO2max was recorded in 57 subjects including both male and female of age group of 18-22 years age group after Queen's college step test. The study showed that $\mathrm{VO} 2 \mathrm{max}$ in obese was $41.3 \pm 8.7 \mathrm{ml} / \mathrm{kg} / \mathrm{min}$ and in non obsess $42.2 \pm 7.9 \mathrm{ml} / \mathrm{kg} / \mathrm{min}$. $^{(7)}$

\section{Conclusion}

The present study showed there is negative correlation between BMI and $\mathrm{VO} 2$ max. VO2max which indicates total body oxygen consumption depends on ventilation and cardiac output. In obesity due to accumulation of fat lungs capacity to increase the ventilation is impaired which affects the VO2max. Obesity also limits the cardiovascular endurance with increase in workload. Therefore daily physical activity in early age of life should be promoted for better performance and to increase the endurance of muscle

\section{Acknowledgment}

We are thankful to the principal cum chief superintendent Assam Medical College and Hospital for kindly allowing us to carry out my research work.

\section{References}

1. Forelicher Victor F., Myers Jonathan :basic of exercise physiology, exercise and heart, 5 th edition, $4 \mathrm{p}$.

2. Cotes J.E: physiology of exercise and changes resulting from lung disease: lung function physiology, measurements and application in medicine, 6th edition, 388p.

3. Guyton and Hall: sports physiology, Text Book Of Medical Physiology, 12th edition, ch 84,1032 p
4. .Shete, A. (2014). A Study of VO 2 Max and Body Fat Percentage in Female Athletes. Journal of Clinical And Diagnostic Research.

5. Shirur, S., L, R. and N, S. (2014). Effect of increased adiposity on cardiorespiratory fitness of young Indian individuals. International Journal of Biomedical Research, 5(11), p.662.

6. Setty, P., Padmanabha, B. and Doddamani, B. (2013). Correlation between obesity and cardio respiratory fitness. International Journal of Medical Science and Public Health, 2(2), p.300.

7. Tauseef, N., Nadeema, R. and Ouber, Q. (2015). Assessment of cardiovascular fitness [VO2 max] among medical students by Queens College step test. International Journal of Biomedical and Advance Research, [online] 6(05), pp.418-421. 